\title{
Gyrométrie interférométrique
}

$>$ Gaël DÉSILLES, Alexandre MARY

THALES Avionics, Châtellerault

$\gg$ Gilles FEUGNET, François GUTTY, Jean-Paul POCHOLLE, Sylvain SCHWARTZ

THALES Research and Technology, Palaiseau Alexandre.Mary@fr.thalesgroup.com

D epuis 40 ans, la technologie de la gyrométrie interférométrique a été développée conjointement au domaine de l'aéronautique. Dans la recherche de capteurs de rotation de plus en plus performants et robustes en toute situation, le gyromètre laser s'est particulièrement démarqué pour ses larges performances, tels sa grande sensibilité et son facteur d'échelle très stable dans le temps, audelà d'un élégant principe de fonctionnement.

Dans cet article, nous proposons d'introduire quelques notions fondamentales et éléments technologiques de la gyrométrie interférométrique. Nous nous placerons pour cela dans le cadre de la relativité restreinte d'Einstein, pour montrer comment intervient un phénomène de désynchronisation relativiste d'horloges mobiles dans un référentiel en rotation, et comment ce phénomène est exploité dans différentes technologies de gyrométrie : le tourniquet aux escargots (décrit seulement en tant qu'expérience de pensée), le gyrolaser à base d'hélium et de néon gazeux, le gyrolaser à cavité résonnante cristalline et, dans un futur plus lointain, le gyromètre à atomes froids. Toutes ces technologies (à l'exception notable de la première) ont été démontrées expérimentalement, que ce soit à l'état de produit fini embarqué, à l'état de prototype ou à l'état d'expérimentation en laboratoire.

\section{De la course d'escargots sur un tourniquet à l'aéronautique moderne}

L'idée d'un capteur de rotation fondé sur des phénomènes optiques est généralement reliée à une expérience considérée comme fondatrice et menée par le physicien français Gérard Sagnac en 1913. Sagnac, expérimentateur de génie, parvint en effet à observer par interférométrie l'effet induit par une rotation sur la différence de phase entre deux faisceaux se propageant en sens contraire à l'intérieur d'une cavité fermée montée sur un plateau rotatif (figure 1a).
Cet effet, proportionnel à la vitesse de rotation, est lié au fait que le temps mis par les faisceaux pour parcourir un tour complet est différent selon le sens de propagation. Comme nous allons le montrer succinctement, il peut s'interpréter comme résultant de l'addition des vitesses en relativité restreinte. En particulier, ce qui est vrai pour ces photons l'est également pour des objets doués de masse et parcourant une trajectoire similaire. Considérons par exemple deux escargots sur un tourniquet, chacun se déplaçant à la même vitesse constante $v^{\prime}$ mais dans un sens différent (figure 2). Supposons que leur trajectoire est la périphérie de rayon $R$ du tourniquet, auquel on a imprimé la vitesse angulaire $\dot{\theta}$ par rapport au référentiel du laboratoire (considéré comme galiléen). Les lois de composition des vitesses en relativité restreinte entraînent, dans le référentiel du laboratoire, des vitesses différentes pour les deux escargots de l'expérience, en raison du rythme de marche différent de deux horloges placées respectivement sur le dos de chaque escargot - un phénomène analogue existe sur les satelittes de la constellation GPS, et il est si intense qu'il doit être compensé pour atteindre la précision décamétrique.

$\gg$

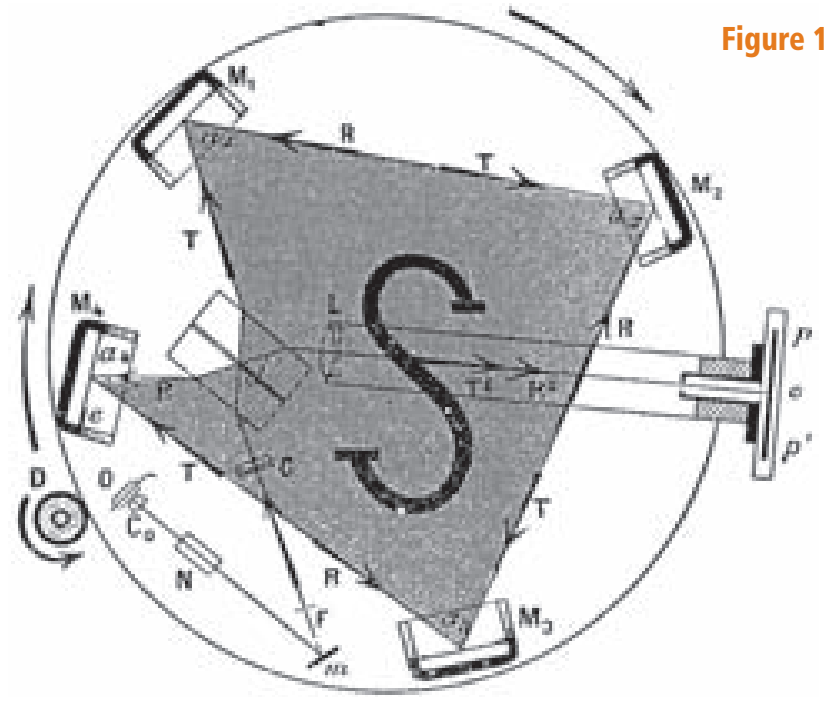

a. Schéma descriptif de l'expérience de G.Sagnac, publié dans son article à l'Académie des Sciences en 1913

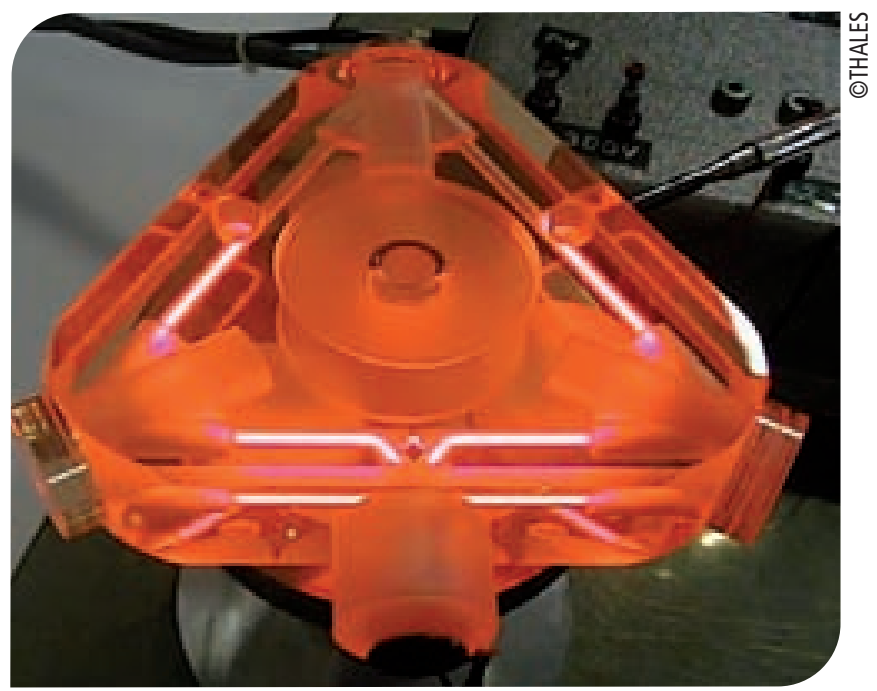

b. Un gyrolaser, aujourd'hui, où l'effet Sagnac se manifeste sur deux ondes laser contrarotatives (ici : cliché du gyrolaser de $33 \mathrm{~cm}$ de THALES Avionics). 


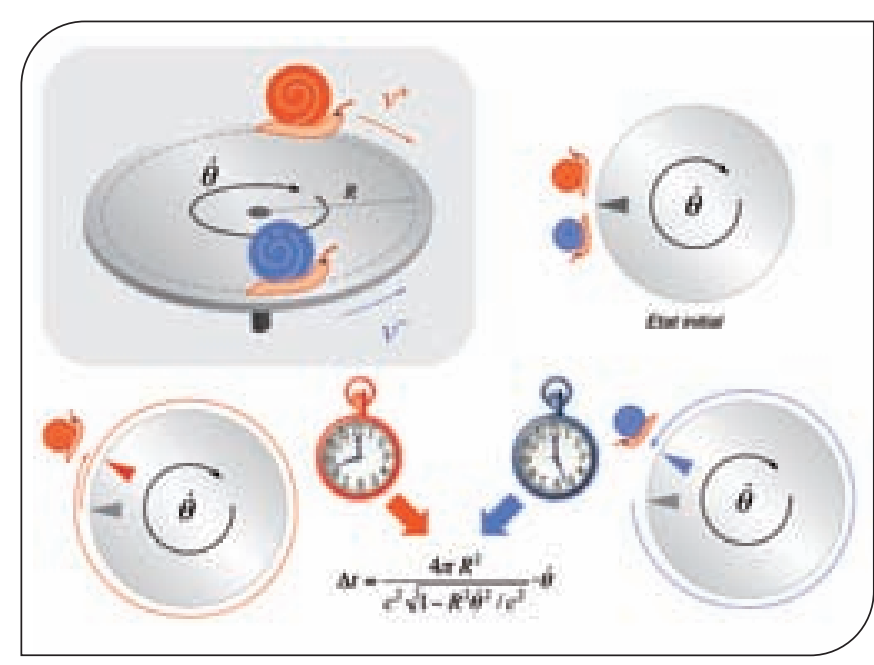

Figure 2. Illustration de l'effet Sagnac sur des escargots à bord d'un tourniquet.

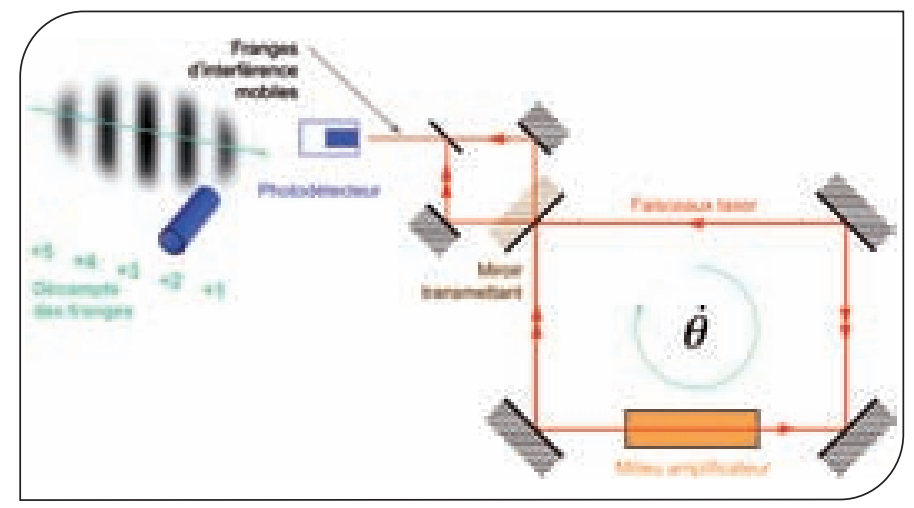

Figure 3. L'effet Sagnac se manifeste sur un gyrolaser, dans lequel deux ondes contrarotatives sont mises en interférence, après avoir parcouru un périmètre optique délimité par des miroirs. Un photodétecteur placé devant les ondes recombinées permet de compter les franges d'interférence pendant chaque unité de temps, ce nombre étant proportionnel à la vitesse de rotation d'entrée $\dot{\theta}$.
Plus précisément, on peut montrer que le temps mis par les deux cobayes de l'expérience pour faire un tour complet du périmètre du tourniquet sera légèrement différent. Cette différence temporelle est donnée dans le référentiel tournant par:

$$
\Delta t=\frac{4 \pi R^{2}}{c^{2} \sqrt{1-R^{2} \dot{\theta}^{2} / c^{2}}} \cdot \dot{\theta}
$$

Dans la limite où $R \dot{\theta} / c<<1$, c'est-à-dire lorsque la vitesse de déplacement du tourniquet en bordure de périmètre est faible devant celle de la lumière, on retrouve l'expression classiquement établie pour l'effet Sagnac:

$$
\Delta t=\frac{4 A}{c^{2}} \cdot \dot{\theta}
$$

où $A$ est l'aire inscrite dans le parcours, et $c$ la célérité de la lumière dans le vide. En pratique, cette différence de temps $s$ 'avère extrêmement faible : de l'ordre de $10^{-21} \mathrm{~s}$ pour un tourniquet d'aire $A \cong 1 \mathrm{~m}^{2}$ et une rotation $\dot{\theta}$ de $10 \%$ (qui est l'ordre de grandeur de la vitesse de rotation de la Terre). Une telle quantité est très difficile à mesurer sur des escargots. De plus, le fait de remplacer les escargots par des photons ne modifie en rien ce bilan : la différence de temps constatée (effet Sagnac) est indépendante de la nature de l'objet considéré, et de sa vitesse de propagation. Sur un parcours circulaire de périmètre $30 \mathrm{~cm}$, cette différence de temps n'est plus que de l'ordre de $10^{-23} \mathrm{~s}$ pour une rotation de $10 \%$ h. On se convaincra aisément des difficultés à mesurer une telle différence de temps, même en présence de rotations beaucoup plus importantes.

En pratique, la différence de temps n'est pas mesurée directement : on utilise plutôt des méthodes interférométriques. On peut alors montrer que la différence de phase induite par l'effet Sagnac sur deux fronts d'onde vaut $\Phi=\omega \cdot \Delta t$ où $\omega$ est la pulsation de l'onde dans le référentiel tournant, et $\Delta t$ le décalage temporel Sagnac.

Même exprimée en termes de déphasage, la sensibilité demeure faible. Dans l'exemple cité plus haut, nous obtiendrions un déphasage $\Phi \approx 1,7.10^{-6} \circ$, pour une vitesse de rotation de $10 \%$, et pour une longueur d'onde $\lambda=633 \mathrm{~nm}$ (soit une pulsation $\omega \approx 3.10^{15} \mathrm{rad} / \mathrm{s}$ ).

Cette faible sensibilité peut être amplifiée de deux manières différentes. Une première manière consiste à multiplier le nombre de tours parcourus par le ront d'onde. Cette technique est utilisée dans les gyromètres à fibre optique, où la sensibilité se trouve directement multipliée par le nombre de tours de fibre le long du périmètre. Par exemple, la sensibilité de $\Delta t$ à la vitesse angulaire d'entrée $\dot{\theta}$ est multipliée par 3000 environ pour une fibre de $1 \mathrm{~km}$ enroulée sur un périmètre de $30 \mathrm{~cm}$ : nous obtenons une différence de temps de l'ordre de $3.10^{-20}$ s pour la même rotation de $10 \%$ (déphasage correspondant pour $\lambda=$ $633 \mathrm{~nm}: \Phi \approx 5.10^{-3}{ }^{\circ}$ ).

Une deuxième manière de compenser la faible sensibilité consiste à installer une résonnance laser sur des photons à l'intérieur d'une cavité. Les photons restant plus longtemps dans la cavité, ils effectuent un grand nombre de tours de cavité avant d'être utilisés pour la lecture du signal.

Dans la suite de cet article, nous allons détailler ce principe sur lequel se base le gyromètre laser.

\section{L'apport du laser à l'effet Sagnac}

Découvert au début des années 1960, l'effet laser a été très rapidement compris comme un moyen d'augmenter la faible sensibilité de l'effet Sagnac.

Considérons une cavité résonnante en anneau, dans laquelle deux faisceaux laser contrarotatifs sont créés à partir d'une décharge électroluminescente dans ungaz (figure 3). La cavité résonnante est constituée de 3 ou 4 miroirs à fort pouvoir réfléchissant, un de ces miroirs étant translatable afin d'ajuster la longueur de la cavité optique pour permettre au laser d'osciller à la fréquence correspondant au maximum de gain du milieu amplificateur.

Si le dispositif est placé en rotation, les deux faisceaux laser sont déphasés de la quantité $\Phi=\omega \cdot \Delta t$ où $\omega$ est la pulsation de l'onde laser dans le référentiel tournant, et $\Delta t$ le décalage temporel lié à l'effet Sagnac. Le déphasage induit $\Phi$ entraîne 
à son tour une différence entre leurs fréquences propres que l'on peut prouver être égale à :

$$
\Delta f=\frac{4 A}{\lambda L} \cdot \dot{\theta}
$$

où $L$ est la longueur totale de la cavité, et $\lambda$ la longueur d'onde moyenne de chaque faisceau.

Cette différence de fréquences est mesurée en recombinant les faisceaux : il apparaît en effet une figure d'interférence mobile, le signal de battement mesuré étant alors directement proportionnel à la vitesse de rotation du dispositif dans l'espace via le terme $K_{1}=4 A /(\lambda . L)$ appelé facteur d'échelle.

Le retard temporel de $10^{-23} \mathrm{~s}$ correspond sur un gyrolaser de $30 \mathrm{~cm}$ de périmètre à une différence de fréquence de $20 \mathrm{~Hz}$, facilement mesurable.

En pratique, la différence de fréquence est intégrée dans le temps en comptant le nombre de franges issues du signal de battement qui défilent sur un détecteur. Un second détecteur est utilisé pour connaître le sens de rotation.

\section{Le phénomène de lock-in - un inconvénient intrinsèque}

Idéalement, la caractéristique du capteur gyrolaser est une fonction linéaire de facteur d'échelle $K_{1}$. Cependant, plusieurs phénomènes conduisent à s'en éloigner. Le plus limitant est le phénomène dit de "lock-in». En dessous d'une certaine vitesse de rotation très faible, le décalage fréquentiel décrit précédemment devient inférieur au couplage entre les deux oscillateurs que sont les faisceaux laser, conduisant à une synchronisation de leur fréquence. Les deux ondes étant synchronisées, la figure d'interférences se fige par rapport aux détecteurs : le gyrolaser renvoie alors la mesure 0 . On parle de gyrolaser "aveugle», d'où également le terme de « zone aveugle » pour désigner le domaine de rotation d'entrée où ce phénomène de verrouillage se produit. En pratique, la zone aveugle est typiquement de l'ordre de $0,1 \%$, c'est-à-dire environ 40000 fois la sensibilité recherchée sur les applications aéronautiques.
La principale origine du couplage provient du phénomène de rétrodiffusion. En effet, sur chacun des miroirs, dont la surface est nécessairement imparfaite, une partie de l'énergie du faisceau incident est diffusée dans le faisceau opposé. Cet échange d'énergie est réciproque entre les deux ondes et se cumule sur tous les miroirs. II en résulte que plus la diffusion des miroirs sera grande, plus le couplage entre les deux faisceaux sera important, et plus la zone aveugle sera étendue. Même fabriqués avec une extrême précision, il existe toujours une faible rétrodiffusion liée à des défauts ou irrégularités de surface, menant à une zone aveugle inacceptable au vu des performances finales recherchées.

Pour s'en affranchir, la solution la plus courante consiste à placer le gyrolaser sur un dispositif mécanique de rotation alternée à moyenne nulle (roue d'activation). L'idée est d'animer en permanence le gyrolaser d'un mouvement d'oscillation angulaire à moyenne nulle autour de son axe sensible, de sorte que la plupart $\gg$

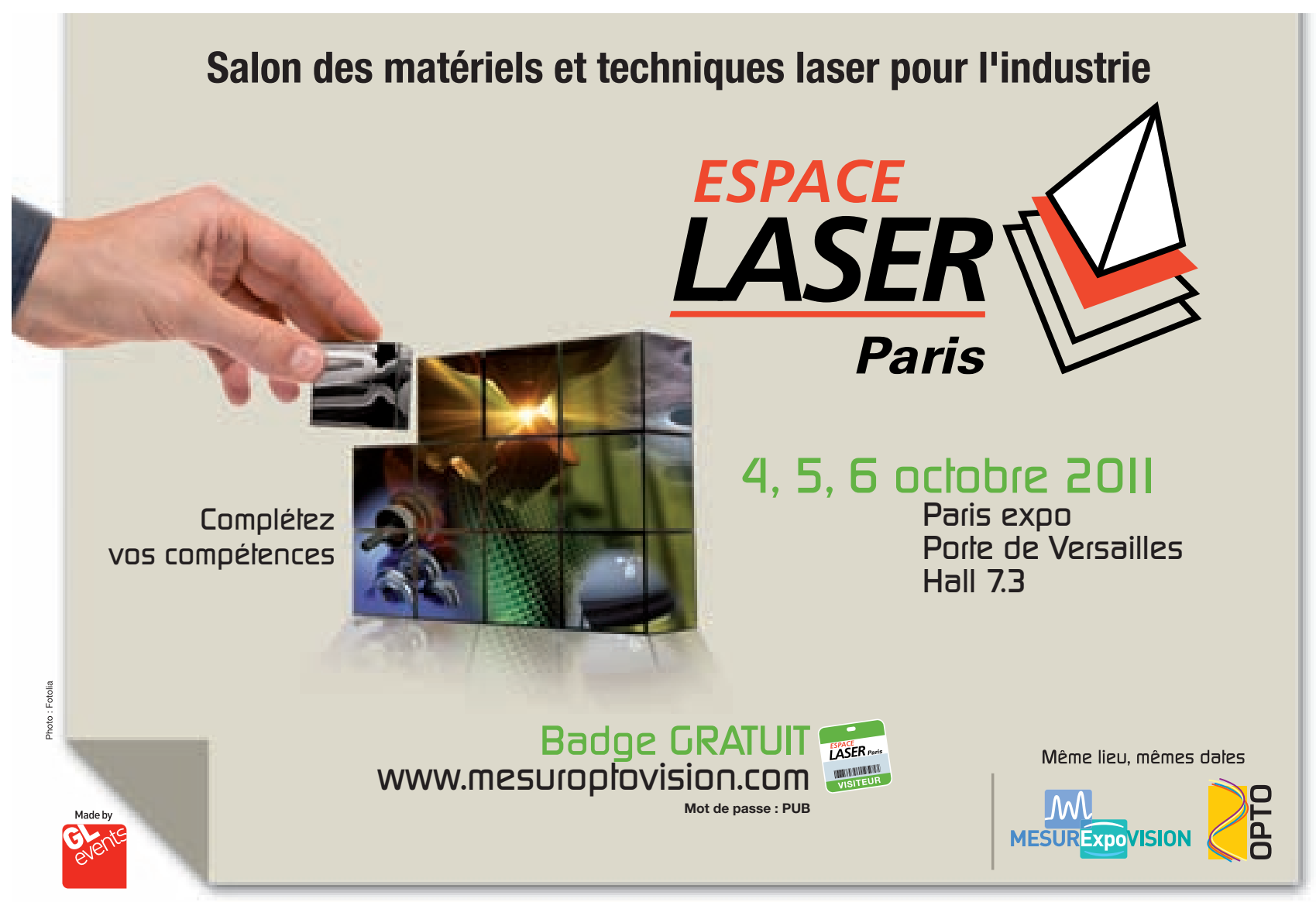


du temps la vitesse de rotation d'entrée possède une valeur au-delà de la zone aveugle. Le gyrolaser restitue donc un signal fidèle la plupart du temps. Toutefois, cette activation sinusoïdale n'est pas suffisante pour assurer de bonnes performances en sortie de capteur, car un nouveau phénomène de synchronisation apparaît aux basses vitesses de rotation. Ce nouveau verrouillage appelé « lock-in dynamique» se traduit par une réponse encore insuffisamment linéaire du capteur à l'intérieur de la zone aveugle ( $f$ gure 4).

Une parade efficace à ce second problème, appelée super-activation, consiste à induire périodiquement sur le gyrolaser une rotation aléatoire d'amplitude limitée (en pratique quelques secondes $d^{\prime}$ arc). La superposition des deux sollicitations (rotation sinusoïdale et rotation aléatoire) linéarise cette fois la réponse du gyrolaser vis-à-vis d'une vitesse de rotation d'entrée du porteur.

Néanmoins cette linéarité de la réponse s'accompagne d'un bruit sur la sortie, spécifique des gyrolasers. En effet, à chaque traversée de la zone aveugle, une erreur statistique sur la phase de l'onde est générée, conduisant à un petit déplacement aléatoire de la figure d'interférences devant les détecteurs. D'une traversée à I'autre, ces petits déplacements statistiquement indépendants induisent tantôt un surplus de translation des franges, tantôt un déficit de translation. À chaque pas de temps, la chaîne de détection comptabilise alors un (petit) nombre aléatoire de déplacements angulaires en plus ou en moins, conduisant à biaiser la mesure en sortie d'une quantité aléatoire limitée.

Si l'on considère l'angle obtenu par intégration temporelle de la sortie du gyromètre, on peut montrer qu'il résulte de ces offsets aléatoires une erreur aléatoire globale dont l'écart-type est égal à :

$$
\sigma_{\Delta \theta}=\frac{\Omega_{L}}{\sqrt{2 \pi K_{1} \Omega_{a}}} \cdot \sqrt{t}
$$

où $\Omega_{L}$ et $\Omega_{a}$ sont respectivement l'amplitude de la zone aveugle et de l'activation. On pourra observer par contre, peutêtre non sans surprise, que l'écart-type ne dépend pas de l'amplitude de la super-

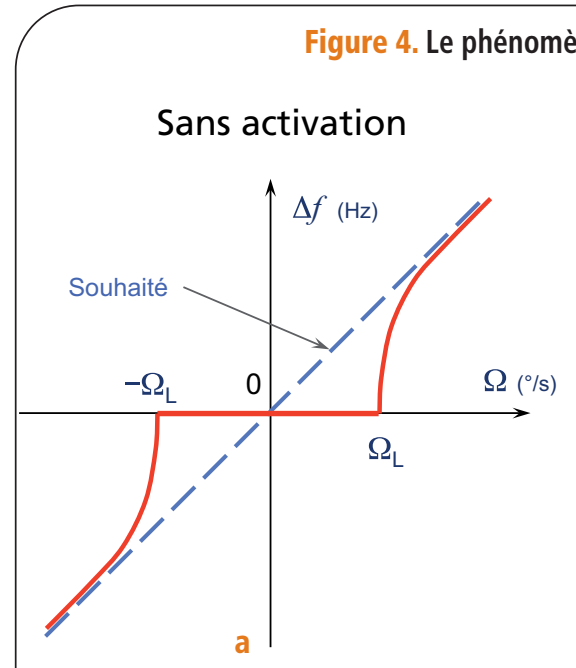

Figure 4. Le phénomène de lock-in dynamique.

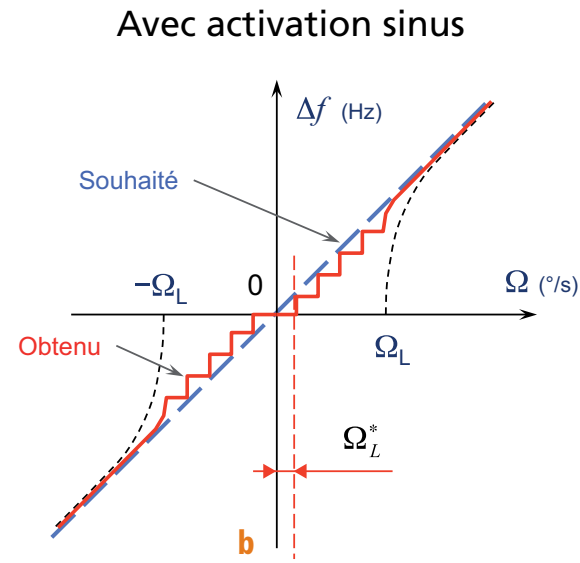

a. Sans activation : la réponse du gyrolaser comporte une "zone aveugle », c'est-à-dire un domaine de vitesses de rotation en entrée $\Omega$ à l'intérieur duquel le capteur renvoie la mesure 0 (absence de battement entre ondes laser).

b. Avec activation purement sinusoïdale : le gyrolaser comporte encore une zone de réponse non linéaire avec de multiples petites sous-zones aveugles locales, de taille $\Omega_{\mathrm{L}}{ }^{*}$.

activation. On diminue donc l'impact de l'erreur en augmentant la taille du gyromètre (augmentation du facteur d'échelle $K_{1}$ ) et en ayant des miroirs à faible diffusion (diminution de $\Omega_{L}$ ). Il n'en demeure pas moins que l'information angulaire "dérive» de façon proportionnelle à la racine carrée du temps, phénomène appelé génériquement « marche au hasard ».

\section{Le gyrolaser à milieu à gain "hélium-néon" ( $\mathrm{He}-\mathrm{Ne}$ )}

Historiquement, le mélange gazeux hélium-néon a été l'un des premiers milieux étudiés pour ses propriétés d'émission stimulée. Dès le début des essais du gyrolaser en laboratoire, un mélange d'hélium et de néon a été utilisé à très faible pression. Rapidement, il a été mis en évidence des problèmes de stabilité de la puissance émise dans les deux faisceaux contra-rotatifs, liés au fait que ces deux faisceaux sont en compétition pour le même milieu amplificateur. Ceci rend le laser instable car plus un mode est intense, plus il va avoir tendance à s'accaparer le gain au détriment de l'autre. La solution consiste à désaccorder volontairement la cavité par rapport au maximum de la courbe de gain. Les atomes qui vont alors participer le plus efficace- ment à l'amplification laser sont ceux dont la vitesse les ramène à résonance (par effet Doppler) avecl'un des modes de la cavité. II s'ensuit que les atomes se déplaçant dans un sens donné (sous l'effet de leur agitation thermique) vont interagir préférentiellement avec l'une des deux ondes plutôt qu'avec l'autre, d'où une diminution du phénomène de compétition, suffisante pour assurer leur coexistence et donc le fonctionnement du dispositif en tant que gyrolaser. De plus, on utilise classiquement un mélange des isotopes 20 et 22 du néon, ce qui crée deux courbes de gain légèrement décalées (d'environ $825 \mathrm{MHz}$ ). On peut alors faire fonctionner le gyrolaser au maximum de la courbe de gain total, tout en étant hors-résonance avec chacun des deux isotopes (figure 5).

\section{Le gyrolaser à état solide}

La totalité des gyrolasers produits actuellement utilisent comme milieu amplificateur un mélange gazeux d'hélium et de néon pompé par décharge électrique, tel que décrit précédemment.

Une possibilité, en cours de développement à Thales, est de remplacer le milieu amplificateur gazeux par un milieu solide, par exemple un cristal de Nd-YAG pompé 


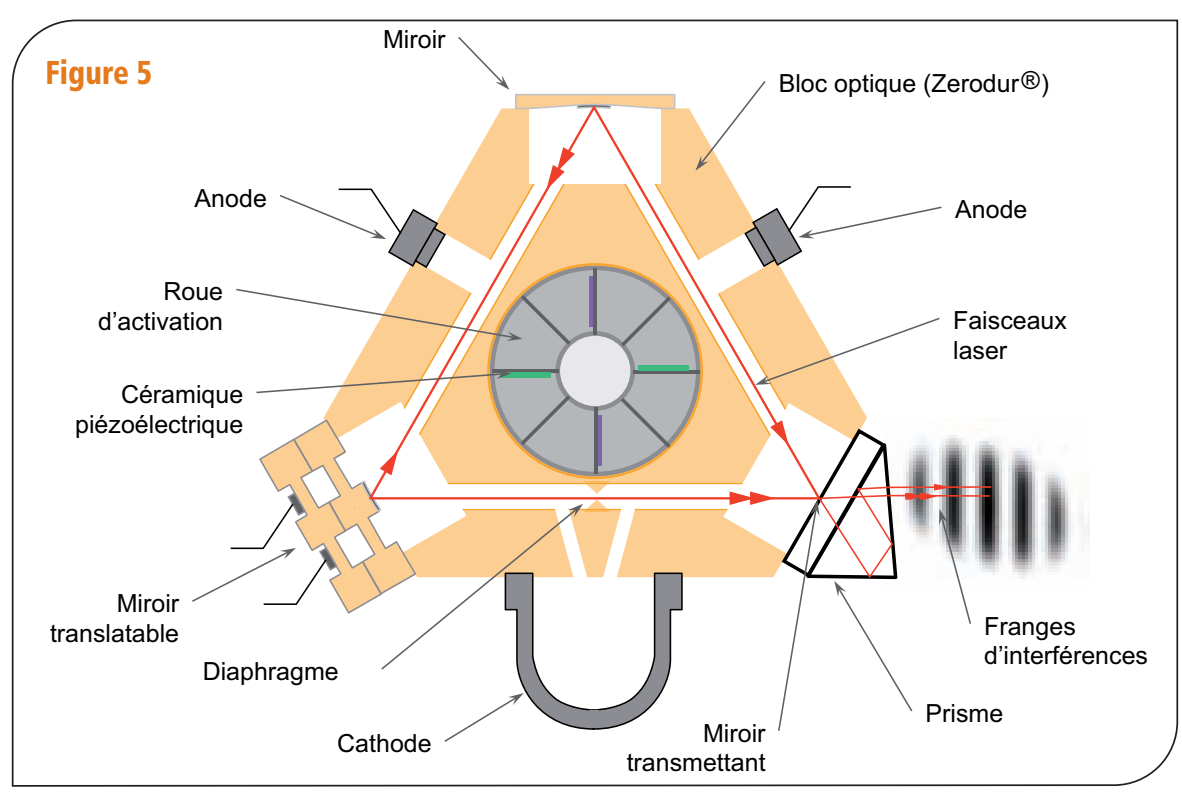

par une diode laser, afin de s'affranchir des contraintes industrielles liées à l'utilisation d'un gaz (figure 6).

Toutefois, une telle substitution n'est pas sans poser quelques difficultés. En effet, le milieu à gain solide que l'on cherche à utiliser est à élargissement homogène, ce qui veut dire que les modes contrarotatifs de la cavité en anneau sont soumis au phénomène de compétition pour le gain. Il en résulte une dynamique très différente de celle du gyrolaser à gaz, a priori mal adaptée à une utilisation en tant que gyrolaser. En particulier, on peut montrer que la présence d'un réseau d'inversion de population induit par émission stimulée tend à empêcher la coexistence des modes contrarotatifs.

Un dispositif additionnel destiné à stabiliser le régime de battement et à permettre ainsi une utilisation du laser en anneau à état solide en tant que gyrolaser peut toutefois être introduit. L'idée est d'infliger à chaque instant, à l'aide d'une boucle de contre-réaction, des pertes dépendantes du sens de propagation et dont la valeur est d'autant plus importante que l'intensité lumineuse du mode correspondant est élevée. Pour générer ces pertes différentielles, on peut agir sur la polarisation des champs, en combinant un miroir polarisant, une cavité non-planaire et un rotateur
Un gyrolaser est réalisé de façon monolithique dans un bloc de vitro-céramique à faible coefficient de dilatation (Zerodur ${ }^{\circledR}$ - SCHOTT), dans lequel une décharge continue de l'ordre de quelques centaines de volts est créée entre une cathode et deux anodes, ces électrodes étant scellées au bloc par une liaison verre-métal. La forme de la cavité peut

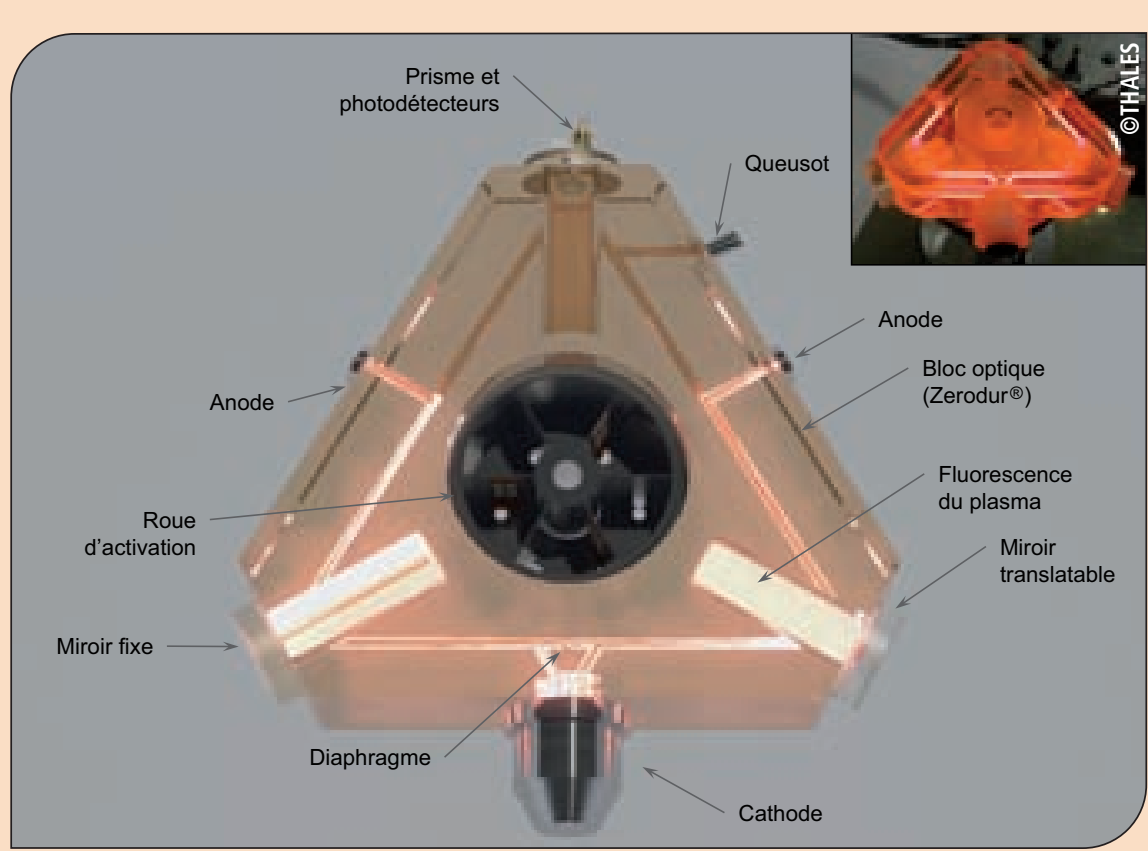

être triangulaire ou carrée. Les miroirs ( 3 ou 4 selon la forme du périmètre) sont liés au bloc par adhérence moléculaire.

Les exigences industrielles et de performances (zone aveugle, faux zéro) entrainent des spécifications extrêmement sévères sur le niveau de transmission, réflexion, absorption et diffusion pour

\section{Technologies du gyrolaser HeNe}

\section{Pour assurer un fonctionnement nomi- nal du laser (stabilité du résonateur et monomodalité) et simplifier l'alignement optique de la cavité, il est nécessaire d'uti- liser au moins un miroir sphérique, et d'in- troduire dans la cavité un diaphragme qui permettra d'éliminer les modes spatiaux autres que le fondamental (monomoda- lité transverse).}

La monomodalité longitudinale du laser est assurée par l'asservissement de la longueur de cavité mentionné précédemment : un des miroirs est translatable. Un transducteur piézo-électrique collé à I'arrière de ce miroir permet de déplacer la partie active du miroir sur une plage de quelques $\mu \mathrm{m}$, suffisante pour compenser les diverses évolutions de la longueur de la cavité générées principalement par les variations thermiques de l'environnement. 


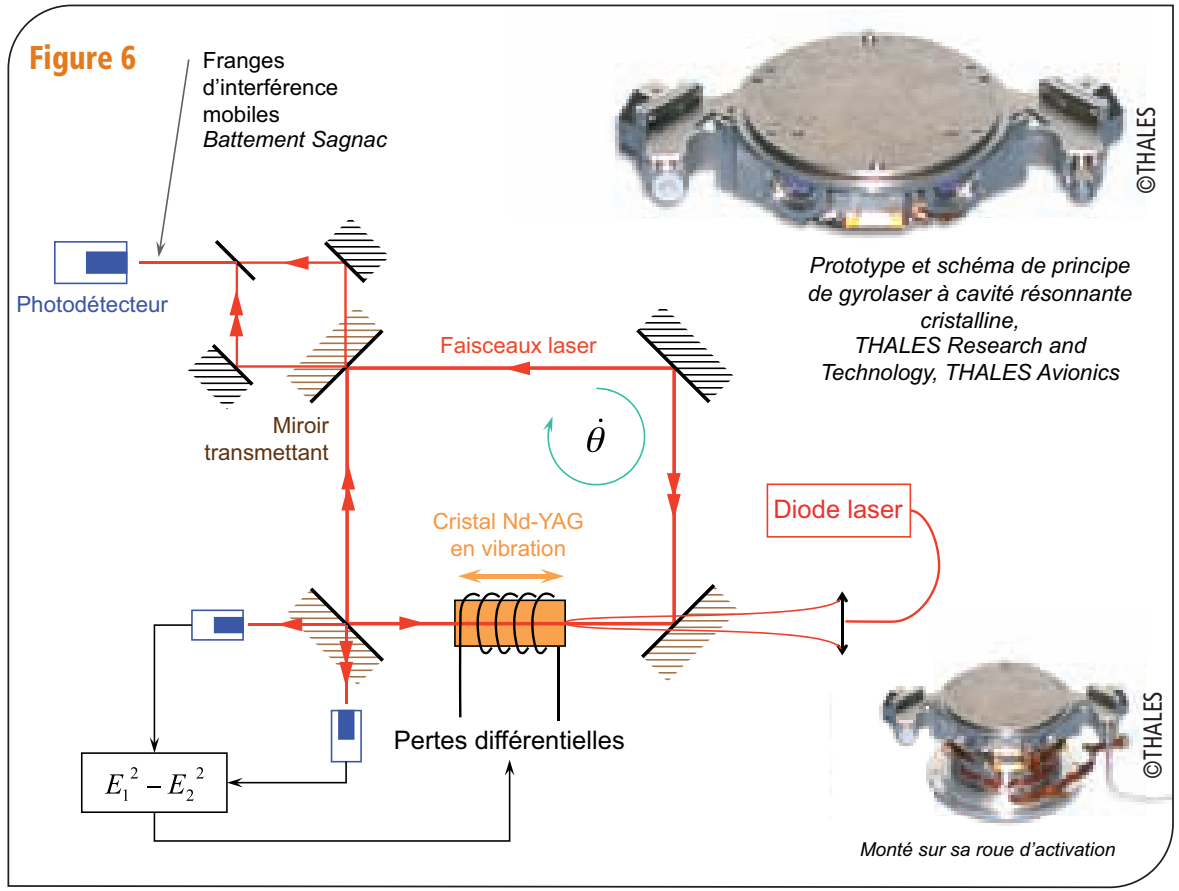

Faraday (ce dernier n'étant autre que le cristal servant de milieu à gain). Ce dispositif permet effectivement de stabiliser le régime de battement au-delà d'une certaine vitesse de rotation, d'où un gyrolaser Nd-YAG pompé diode et à fonctionnement continu. Un point important réside dans le fait que le dispositif permettant de créer, au sein de la cavité laser, des pertes contrôlables et dépendantes du sens de propagation, ne nécessite pas I'introduction d'élément supplémentaire dans la cavité laser bien qu'il repose sur la combinaison de trois effets optiques successifs. Cet aspect du dispositif est primordial d'un point de vue industriel. Toutefois, les performances du gyrolaser ainsi obtenues se révèlent insuffisantes du point de vue d'une utilisation pratique, car la réponse en fréquence est perturbée par les couplages non-linéaires induits par la présence du réseau de gain dans le cristal de Nd-YAG. Pour s'affranchir de ce problème, I'idée consiste à faire vibrer le milieu à gain le long de l'axe de la cavité optique, avec une amplitude telle que chacun des atomes ne soit plus confiné dans un nœud ou un ventre de l'onde stationnaire optique, mais voie au contraire en moyenne la même intensité que tous ses voisins. La fréquence de vibration doit également être suffisamment grande pour que les atomes n'aient pas le temps de suivre les variations d'intensité qui en résultent, et ne soient sensibles à l'intensité moyenne (le temps de réponse typique du milieu à gain utilisé est de l'ordre de 200 ms). Nous avons pu démontrer expérimentalement qu'en présence d'un tel dispositif, le gyrolaser à état solide avait un comportement similaire à celui d'un gyrolaser à hélium-néon, avec une zone aveugle autour de zéro dont la taille est déterminée par la qualité des miroirs utilisés. Un prototype à été réalisé, dont les performances sont actuellement en cours de caractérisation (voir photo de la figure 6).

\section{Vers de nouveaux types de gyromètres}

Plus de 30 ans après sa mise en service, le gyrolaser reste un capteur inertiel de tout premier plan pour les applications de haute et moyenne performance, notamment grâce à ses très bonnes propriétés de stabilité long terme et de dynamique de mesure. Le remplacement du milieu amplificateur gazeux par un milieu solide, en cours de développement à Thales, pourrait constituer à moyen terme une rupture significative en termes de coût et de durée de vie pour ce type de capteur. Enfin, le principe de l'effet Sagnac et de la gyrométrie interférométrique peut

\section{les limitateurs de performance} des gyrolasers $\mathrm{He}-\mathrm{Ne}$

- Le faux-zéro

Le "biais » ou "faux-zéro » est la mesure délivrée par le capteur lorsque la vitesse d'entrée est nulle. Cette erreur n'est pas gênante en soi si elle est stable, car elle peut être compensée par un calculateur. Le faux-zéro trouve son origine physique dans les dissymétries entre les deux ondes contrarotatives. Cette analyse amène à systématiser le principe de symétrie dans toute la conception de la cavité, ce qui conduit aux choix suivants (dans le cas d'une cavité triangulaire) :

- une symétrie parfaite des décharges ;

- une symétrie associée de la cavité avec la mise en place du miroir sphérique à l'opposé de la cathode ;

- l'usinage du diaphragme sur l'axe cathode-miroir sphérique ;

- un dimensionnement rigoureusement identique de part et d'autre de ce même axe, aussi bien pour les parties actives des capillaires (zones à plasma) que non actives.

Il est important de noter ici que le plan des faisceaux optiques doit être confondu au mieux avec celui déterminé par les capillaires, toujours par souci de symétrie. Cela induit des tolérances serrées, en particulier sur l'angle des faces " miroirs » du bloc avec le plan des capillaires. À titre d'illustration, sur un gyromètre triangulaire de $33 \mathrm{~cm}$ de périmètre, une erreur angulaire de 1 seconde $d^{\prime}$ arc sur l'inclinaison d'un miroir décentre le faisceau de $25 \mu \mathrm{m}$ !...

L'ensemble des principes précédents étant respecté, le faux-zéro résiduel mesuré classiquement est inférieur à $0,005 \%$ h (périmètre $33 \mathrm{~cm}$ ).

\section{- Le facteur d'échelle}

La stabilité et la linéarité sur le domaine de mesure ( 0 à $200 \%$ voire $400 \%$ typiquement pour les applications avioniques civiles) constituent les critères importants pour le facteur d'échelle. Les performances obtenues dépendent de la stabilité dimensionnelle de la cavité, des évolutions des caractéristiques du laser ainsi que de la diffusion des miroirs. La maîtrise de ces paramètres passe donc par l'utilisation de matériau à très faible coefficient de dilatation comme le Zerodur ${ }^{\circledR}$, ainsi que par une optimisation des technologies de conditionnement du laser (absence de pollutions, de fuites, stabilisation des paramètres électriques de la décharge, etc.).

Moyennant ces choix, on atteint aisément une stabilité et une linéarité de facteur d'échelle de l'ordre de quelques parties par million. 
être étendu au-delà des photons, dans le cadre de l'interférométrie atomique qui repose sur les fonctions d'ondes associées aux atomes par la mécanique quantique. Les gyromètres atomiques, qui présentent un gain de sensibilité intrinsèque d'environ 11 ordres de grandeur par rapport aux gyromètres optiques, ont déjà été démontrés dans certains laboratoires académiques en utilisant des atomes refroidis par laser. Des travaux sont actuellement en cours à Thales autour de la miniaturisation de ces technologies dites « d'atomes froids " pour permettre leur intégration dans les futurs gyromètres, gravimètres et accéléromètres.

Remerciements : Merci à Rémi Andréoletti (THALES Avionics, Châtellerault) pour la réalisation des illustrations 3D.

\section{Le gyrolaser tri-axe PIXYZ}

THALES AVIONICS est I'un des deux seuls fabricants au monde à disposer d'une technologie tri-axe de gyrolaser, c'està-dire un capteur capable de fournir directement une mesure de la vitesse de rotation instantanée tridimensionnelle. Ce capteur, appelé $P I X Y Z$, a été décliné en deux versions : la version PIXYZ22 de $22 \mathrm{~cm}$ de périmètre de cavité, destinée aux applications de haute performance (besoin de précision à $0,01^{\circ} / \mathrm{h}$ ) et la version PIXYZ14, très compacte, dont le périmètre de cavité mesure $14 \mathrm{~cm}$, et destinée à des applications de moyenne performance (besoin de précision de l'ordre de $0,1^{\circ} / \mathrm{h}$ ).

Le principe d'architecture du PIXYZ repose sur trois cavités laser carrées, disposées orthogonalement les unes aux autres de façon à ce que chaque miroir soit commun à exactement deux cavités. La géométrie des cavités est de ce fait évidemment très complexe.

Comparé à un ensemble de 3 gyrolasers mono-axiaux qu'il remplace, le gyrolaser tri-axe PIXYZ présente de nombreux avantages parmi lesquels :

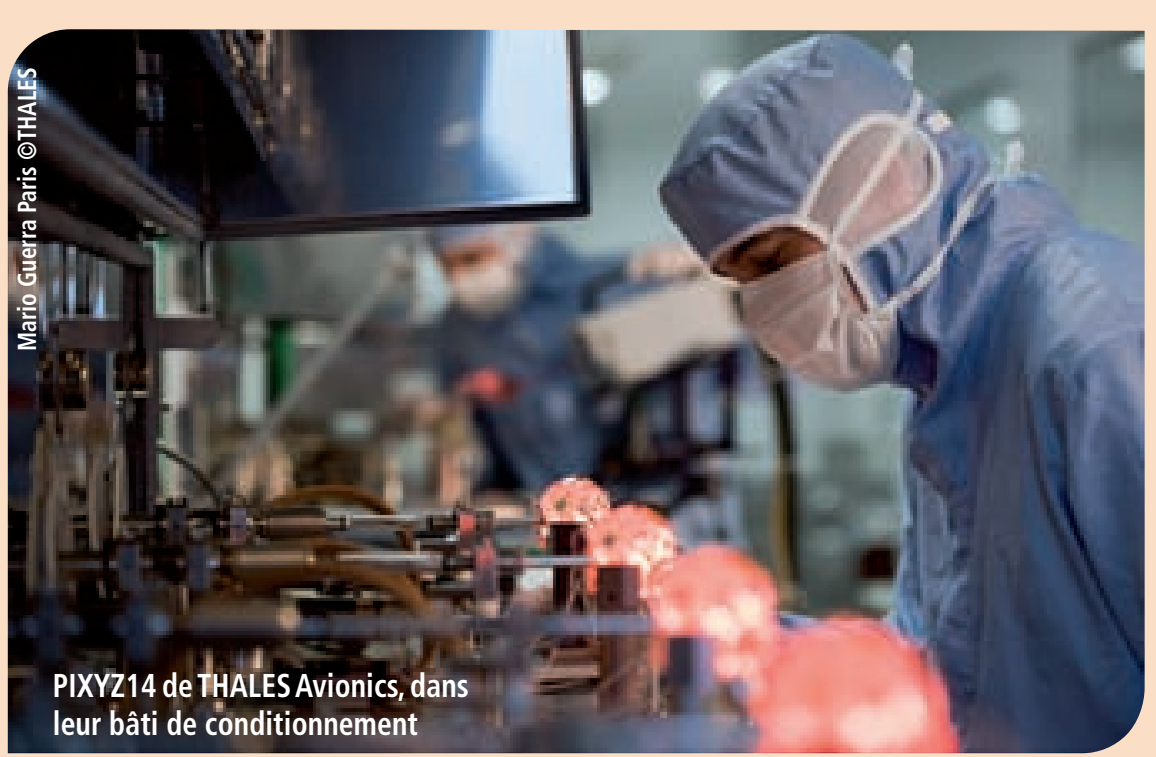

- un nombre réduit de composants (6 miroirs au lieu de 9 ou 12, 7 électrodes au lieu de 9, 1 seul dispositif d'activation commun à tous les axes, au lieu de 3 ) ;

- une activation unique des 3 axes, permettant une réduction de l'impact de la marche au hasard ;

- une meilleure compacité ;

- une meilleure stabilité angulaire entre les 3 voies de mesure ;

- un coût largement inférieur.

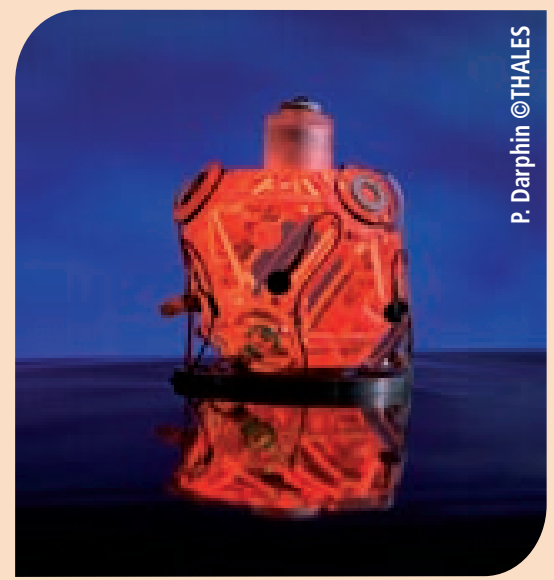

Gyrolaser PIXYZ22 de THALES Avionics

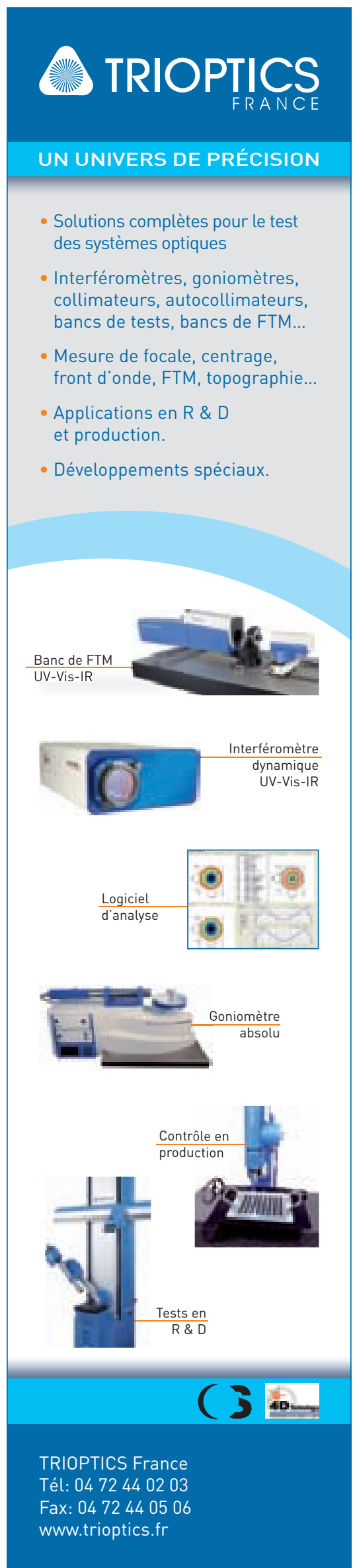

